\title{
A neurotropic herpesvirus infecting the gastropod, abalone, shares ancestry with oyster herpesvirus and a herpesvirus associated with the amphioxus genome
}

\author{
Keith W Savin ${ }^{1 *}$, Benjamin G Cocks ${ }^{1,5}$, Frank Wong ${ }^{2,3}$, Tim Sawbridge ${ }^{1,5}$, Noel Cogan ${ }^{1}$, David Savage ${ }^{1,4}$, \\ Simone Warner ${ }^{2}$
}

\begin{abstract}
Background: With the exception of the oyster herpesvirus OsHV-1, all herpesviruses characterized thus far infect only vertebrates. Some cause neurological disease in their hosts, while others replicate or become latent in neurological tissues. Recently a new herpesvirus causing ganglioneuritis in abalone, a gastropod, was discovered. Molecular analysis of new herpesviruses, such as this one and others, still to be discovered in invertebrates, will provide insight into the evolution of herpesviruses.
\end{abstract}

Results: We sequenced the genome of a neurotropic virus linked to a fatal ganglioneuritis devastating parts of a valuable wild abalone fishery in Australia. We show that the newly identified virus forms part of an ancient clade with its nearest relatives being a herpesvirus infecting bivalves (oyster) and, unexpectedly, one we identified, from published data, apparently integrated within the genome of amphioxus, an invertebrate chordate. Predicted protein sequences from the abalone virus genome have significant similarity to several herpesvirus proteins including the DNA packaging ATPase subunit of (putative) terminase and DNA polymerase. Conservation of amino acid sequences in the terminase across all herpesviruses and phylogenetic analysis using the DNA polymerase and terminase proteins demonstrate that the herpesviruses infecting the molluscs, oyster and abalone, are distantly related. The terminase and polymerase protein sequences from the putative amphioxus herpesvirus share more sequence similarity with those of the mollusc viruses than with sequences from any of the vertebrate herpesviruses analysed.

Conclusions: A family of mollusc herpesviruses, Malacoherpesviridae, that was based on a single virus infecting oyster can now be further established by including a distantly related herpesvirus infecting abalone, which, like many vertebrate viruses is neurotropic. The genome of Branchiostoma floridae (amphioxus) provides evidence for the existence of a herpesvirus associated with this invertebrate chordate. The virus which likely infected amphioxus is, by molecular phylogenetic analysis, more closely related to the other 2 invertebrate viruses than to herpesviruses infecting vertebrates (ie chordates).

\section{Findings}

In 2005 there was an outbreak of acute ganglioneuritis in an Australian population of the edible gastropod mollusc, abalone or Haliotis spp[1]. Using transmission electron microscopy, herpes-like particles were observed in

\footnotetext{
* Correspondence: keith.savin@dpi.vic.gov.au

'Biosciences Research Division, Department of Primary Industries, 1 Park

Drive, Bundoora, Victoria 3083, Australia

Full list of author information is available at the end of the article
}

ganglia of affected abalone[2] and purified virions from moribund abalone nervous tissues were identified as resembling those of herpesviruses, having an icosohedral capsid approximately $100 \mathrm{~nm}$ in diameter surrounded by a $150 \mathrm{~nm}$ diameter spiked envelope[3]. Potential herpesvirus particles were also identified previously in Taiwan following mortalities in Haliotis diversicolor [4]. Recently a diagnostic PCR test has been developed to detect the abalone virus [5]. The test has detected viral DNA 
sequences in diseased abalone from separate geographical locations in Australia and in DNA isolated from a herpes-like virus found some time ago in Taiwan[4].

Three herpesvirus families have been described in the order Herpesvirales - the Herpesviridae which infect Mammalia, Aves and Reptilia, the Alloherpesviridae infecting Amphibia and Osteichthyes (bony fish), and the mollusc-infecting Malacoherpesviridae containing a single virus that infects an invertebrate class, Bivalvia [6-8]. The phylogenetic relationships of these herpesviruses have been well studied and their evolution over epochs is largely synchronous with host lineages $[7,8]$. Gastropods separated early in the Cambrian period from all other known herpesvirus hosts. This unique evolutionary positioning[6] combined with our discovery of a related herpesvirus genome apparently integrated into the genome of another invertebrate, amphioxus, expands the Herpesvirales order and provides two key links to understanding the nature of the ancient ancestors of mollusc and vertebrate herpesviruses. To understand the structural and evolutionary relationships of the abalone virus to other herpesviruses, we purified abalone virus particles and isolated and sequenced genomic DNA using methods previously described[3,9]. The DNA was subjected to multiple displacement amplification[10] and sequenced using the Roche 454 GS-FLX system followed by partial genome assembly using the Newbler algorithm (Roche).

Based on the assembled DNA sequences of the abalone virus, several protein coding sequences predicted using Artemis[11] showed varying distant homology to herpesvirus proteins, most notably those of Ostreid herpesvirus 1 (oyster herpesvirus 1, OsHV-1), a virus infecting bivalve mollusc species[12,13]. BLAST analysis[14] of assembled sequence contigs based on predicted proteins identified 39 full length homologues of OsHV-1 genes (Table 1). These coding sequences, within partial genome scaffold sequences, or as individual coding sequences, have been submitted to Genbank. None of the coding sequences identified appear to be split by introns. Full-length sequences encoding homologues of DNA polymerase and the DNA packaging ATPase subunit of the (putative) terminase (henceforth referred to as the polymerase and terminase respectively), were identified and chosen for use in sequence alignments and phylogenetic analysis (Figures 1 \&2). Hereafter, we will refer to the new abalone virus as abalone herpesvirus or AbHV-1.

During the search for homologues of predicted AbHV1 proteins using BLAST we identified, in the non-redundant (nr) Genbank protein sequence database, Branchiostoma floridae (amphioxus) coding sequences with significant homology to some of those in AbHV-1. The genome of amphioxus has been recently sequenced [15] although final assembly of chromosomes is not yet complete. On further analysis of amphioxus coding sequences using BLASTP with the predicted protein sequences of the oyster herpesvirus OsHV-1 genome (Genbank NC_005881), we identified 19 herpesvirus gene homologues. Consistent with this being an integrated virus, we found that 18 of these genes are clustered within a $150 \mathrm{~kb}$ region of a single amphioxus scaffold BRAFLscaffold_217, including the herpesvirus specific terminase gene[16] and all but 4 of these genes do not contain introns. These virus coding sequences appear to be legitimately assembled within published genome sequence scaffolds and are therefore probably integrated within the amphioxus genome. Further experiments such as fluorescence in situ hybridisation of chromosomes would confirm this. The 19 coding sequences identified are listed in Table 2 along with their OsHV-1 homologues and BLAST scores. We utilised the amphioxus virus terminase and polymerase protein sequence homologues in our analyses.

The putative terminase, or DNA packaging ATPase, appears specific to herpesviruses and some bacteriophages, such as T4[16] and is thought to be an enzyme motor involved in packaging viral DNA into preformed capsids[17]. We used the ATPase motif from this protein to investigate the phylogeny of the herpesviruses.

The ATP hydrolase (ATPase) motif sequences from 20 of the 34 terminase proteins listed in Table 3, plus their T4 bacteriophage homologue and the amphioxus terminase homologue (XP_002591195.1, listed in Table 2), were identified using Interproscan[18] and aligned using ClustalW[19]. Figure 1 shows that 12 amino acids are conserved across all herpesvirus ATPase domain sequences, including those from the abalone, oyster and amphioxus virus genomes, indicating the placement of the abalone virus and putative amphioxus virus within the Herpesvirales order. A common ancestral origin for the mollusc and amphioxus viruses is confirmed by the absence of introns in the terminase gene and the presence of additional amino acid loops (Figure 1). Although being in the same clade (Figure 2), at a protein sequence level the mollusc viruses are only moderately related with $40 \%$ amino acid identity in this conserved viral protein, across their full length.

The phylogenetic analysis comparing concatenated polymerase and terminase full-length proteins (Figure 2, Table 3), illustrates the evolutionary relationships within the Herpesvirales order. The five Alloherpesviridae viruses are clustered together, with separate clades for frog and fish viruses as found previously [8], and the Herpesviridae are clustered into separate major clades reflecting their taxonomic groupings of alpha-, beta- and gammaherpesvirinae sub-families. The phylogenetic analysis confirms a relationship between the amphioxus 
Table 1 OsHV-1 homologues of AbHV-1 coding sequences

\begin{tabular}{|c|c|c|c|c|c|c|}
\hline \multicolumn{2}{|c|}{ AbHV-1 } & \multicolumn{2}{|c|}{ OsHV-1 } & \multicolumn{3}{|c|}{ BLASTP result } \\
\hline Gene & Genbank & Genbank & Description & Ident. & Score & E value \\
\hline AbHVp002c & ADJ95315.1 & YP_024647.1 & ORF109 terminase & $42 \%$ & 620 & $3 e-175$ \\
\hline AbHVp003 & ADL16651.1 & YP_024565.1 & ORF20 RNR2 & $37 \%$ & 252 & $1 e-64$ \\
\hline AbHVp005c & ADL16652.1 & YP_024602.1 & ORF59 & $24 \%$ & 90 & $1 e-15$ \\
\hline AbHVp006 & ADL16653.1 & YP_024573.1 & ORF28 & $26 \%$ & 239 & $2 e-60$ \\
\hline AbHVp013c & ADL16656.1 & YP_024591.1 & ORF47 & $27 \%$ & 336 & 1e-89 \\
\hline AbHVp018c & ADL16657.1 & YP_024590.1 & ORF46 & $31 \%$ & 48 & $6 e-04$ \\
\hline AbHVp019c & ADL16658.1 & $\begin{array}{l}\text { YP_024552.1 } \\
\text { YP_024552.1 }\end{array}$ & $\begin{array}{c}\text { ORF49, ORF7 } \\
\text { primase/helicase }\end{array}$ & $24 \%, 24 \%$ & 94,74 & $5 e-17,1 e-10$ \\
\hline AbHVp024 & ADL16662.1 & YP_024567.1 & ORF22 & $23 \%$ & 234 & $7 e-59$ \\
\hline AbHVp031c & ADL16665.1 & YP_024606.1 & ORF66 & $27 \%$ & 375 & $2 e-101$ \\
\hline AbHVp032 & ADL16666.1 & YP_024607.1 & ORF67 & $32 \%$ & 247 & $3 e-63$ \\
\hline AbHVp034 & ADL16667.1 & YP_024575.1 & ORF30 & $27 \%$ & 53 & $3 e-05$ \\
\hline AbHVp037c & ADL16668.1 & YP_024616.1 & ORF77 & $23 \%$ & 170 & $1 e-39$ \\
\hline AbHVp038c & ADL16669.1 & YP_024587.1 & ORF43 & $27 \%$ & 70 & $1 e-10$ \\
\hline AbHVp039c & ADL16670.1 & YP_024634.1 & ORF95 & $27 \%$ & 94 & $2 e-17$ \\
\hline AbHVp043c & ADL16671.1 & YP_024611.1 & ORF71 & $23 \%$ & 108 & $1 e-21$ \\
\hline AbHVp045c & ADL16672.1 & YP_024604.1 & ORF61 & $29 \%$ & 185 & $1 e-44$ \\
\hline \# AbHVp050 & ADL16674.1 & $\begin{array}{l}\text { YP_024593.1, } \\
\text { YP_024552.1 }\end{array}$ & $\begin{array}{l}\text { ORF49, ORF7 } \\
\text { primase/helicase }\end{array}$ & $21 \% 20 \%$ & 125,90 & $4 e-26,1 e-15$ \\
\hline AbHVp057c & ADJ95314.1 & YP_024639.1 & $\begin{array}{l}\text { ORF100 DNA } \\
\text { polymerase }\end{array}$ & $31 \%$ & 673 & 0.0 \\
\hline AbHVp064 & HQ400676 & YP_024619.1 & ORF80 & & 38.5 & 0.29 \\
\hline AbHVp070c & HQ400677 & YP_024651.1 & ORF113 & $25 \%$ & 105 & $8 e-21$ \\
\hline AbHVp073c & HQ400678 & YP_024650.1 & ORF112 & $26 \%$ & 119 & $7 e-25$ \\
\hline AbHVp075 & HQ400679 & YP_024649.1 & ORF111 & $32 \%$ & 198 & $6 e-49$ \\
\hline AbHVp086 & HQ400681 & YP_024645.1 & ORF107 & $26 \%$ & 134 & $4 e-29$ \\
\hline AbHVp093 & HQ400682 & YP_024622.1 & ORF83 & $19 \%$ & 49 & $3 e-04$ \\
\hline AbHVp102 & HQ400683 & YP_024630.1 & ORF91 & $30 \%$ & 151 & $1 e-34$ \\
\hline AbHVp104c & HQ400684 & YP_024584.1 & ORF40 & $30 \%$ & 238 & $2 e-60$ \\
\hline AbHVp110 & HQ400685 & YP_024595.1 & ORF52 & $34 \%$ & 68 & $4 e-10$ \\
\hline AbHVp111 & HQ400686 & YP_024596.1 & ORF53 & $23 \%$ & 50 & $9 e-04$ \\
\hline AbHVp112 & HQ400687 & $\begin{array}{l}\text { YP_024597.1, } \\
\text { YP_024608.1 }\end{array}$ & ORF54, ORF68 & $43 \%$ & 643 & 0.0 \\
\hline AbHVp113c & HQ400688 & YP_024657.1 & ORF115 & $32 \%$ & 80 & $3 e-13$ \\
\hline AbHVp117c & HQ400689 & YP_024635.1 & ORF96 & $23 \%$ & 53 & $4 e-05$ \\
\hline AbHVp121 & HQ400690 & YP_024633.1 & ORF94 & $28 \%$ & 114 & $2 e-23$ \\
\hline AbHVp130c & HQ400691 & YP_024605.1 & ORF64 & $36 \%$ & 212 & $6 e-53$ \\
\hline AbHVp131 & HQ400692 & YP_024615.1 & ORF76 & $29 \%$ & 202 & $1 e-59$ \\
\hline AbHVp133 & HQ400693 & YP_024569.1 & ORF24 & $23 \%$ & 67 & $3 e-09$ \\
\hline AbHVp134c & HQ400694 & $\begin{array}{l}\text { YP_024608.1, } \\
\text { YP_024597.1 }\end{array}$ & ORF68, ORF54 & $53 \%$ & 784 & 0.0 \\
\hline AbHVp135c & HQ400695 & YP_024624.1 & ORF85 & $26 \%$ & 225 & $1 e-56$ \\
\hline AbHVp136c & HQ400696 & YP_024588.1 & ORF44 & $32 \%$ & 134 & $1 e-29$ \\
\hline AbHVp137 & HQ400697 & YP_024609.1 & ORF69 & $29 \%$ & 172 & $7 e-41$ \\
\hline
\end{tabular}

Note: OsHV ORF49 \& ORF7 are members of a gene family comprising ORF49, ORF7 \& ORF115 OsHV ORF54 \& ORF68 comprise a gene family. AbHV Genbank accessions beginning with "AD" can also be found in scaffold sequences [Genbank:HM631981, Genbank:HM631982]. 


\begin{tabular}{|c|c|}
\hline & \\
\hline & \\
\hline abalone_herpesvirus & IK \\
\hline Ostreid_herpesvirus_1 & GER LKKN I SGY I I PRRCGKS S F SGCM I AL VMAMCP SAG IKC L YTA HKKNQCTDMY S SVEKHV VAL IKEF NR INEAK F L - \\
\hline Ictalurid_herpesvirus_1 & FDE YGKNY TLALAPRQCGK T T IMVI L LAAM I SYL - - - D I E I VVQAQHK TMCE T L YDR VE L VL HE I QHS PWYPEENR I V- \\
\hline Cyprinid_herpesvirus 3 & FNY YNKC YMVATVP RRCGK T T I VSVV L GAML S FM - - - G ID I MVQAQNQNMAST I RKNVQ T FMDAYRDK TWF PDKF K YK - \\
\hline Ranid_herpesvirus_2 & FNVYAKPY TAATVPRRCGKS T IMAI I I SAA IMF L - - - E ID I VIQAHRKE TCL T F SNK I R TYVEQLQTR PWFPSEFR I H - \\
\hline Ranid_herpesvirus_1 & CA I Y EKK Y TAA I VP RRCGK S TML E I I MAG A A L F L - - E IDV L IQA HRNP T CLAL YNRMEQR V GAMQSAPWF PKDFK I T - \\
\hline Murid_herpesvirus_4 & LL T F LQKS PAFL I PRRHGK TW I VTA I I SML L T SV - - DDLH I GYVAHQKHVSLAVF L E I SN I L LAWF PRK N ID I KKE NG - \\
\hline Ovine_herpesvirus_2 & LEV F KQKA SVF L I PRRHGK T W I VVA I I S I L L A S V - - QDLR I GYVA HQKHVANAV F T E V I N T L HAF F PGR Y I DVKKE NG - \\
\hline Human_herpesvirus_8 & LQT F KQKA SVFL I P RRHGK TW I VVA I I SML L A SV - - EN IN I GYVAHQKHVANS VF AE I I K T L CRWF P PK NLN I KKE NG - \\
\hline Panine_herpesvirus_2 & IE I FKQKA TVFLVPRRHGK TWF I IP I IS F L L KHM - - IG IS I GYVAHQKHVSQF VLKE VE FRCRYT FARDYL - VENKDN- \\
\hline Gallid_herpesvirus_1 & IQHFRQR T TVFLVPRRHGK TWF L VPL I SL L VSS F - - EGIR I GYTAHLRKATEPVF I E I F TRL YKWFGAKQVEQVKGE - - \\
\hline erpesvirus_1 & LHH F KQR T SVF LVPRRHGK TWF L VP L I S L L V SCF - - EGLR I GYTAHLRKA TKPVF E E I YAR L CRWYGEDR \\
\hline Meleagrid_herpesvirus_1 & TRHF RQR V SVFLVP RRHGK TWF LVPL I AL AMA S F - - EGIR I GYT SHIRKA IEPVFE E I GDRL RKWFGGH R VDHVKGE - - \\
\hline Suid_he & VRHFRQR V TVFLVPRRHGK TWF LVPL I ALALATF- - RGIRVGYTAHIRKATEPVFEE I HARL RRWCRDAR \\
\hline Macacine_herpesvirus_1 & VR HF RQRA TVFLVPRRHGK T WF I VPL I AL S L A S F - -RGIK I GYTAH IRKATEPVFDE I DACL QGWI \\
\hline Papine_herpesvirus_2 2 & VRH F RQRA TVF LVPRRHGK TWF I VP L I AL S L A S F - - RG IK I GYTAH IRKATEPVFDE I DACL QGWFGS R VDHVKGE - - \\
\hline Cercs & VRHFRQRA TVFLVPRRHGK TWF I VPL I AL S L ASF - - RGIK I GYTAHIRKATEPVFDE I DACL RGWFGAA \\
\hline Human_herpesvirus_1 & VRHFRQRA TVFLVPRRHGK TWF L VPL IALSLASF - - RGIK I GYTAHIRKATEPVFEE I DACL RGWFGSAR VDHVKGE - - \\
\hline Bovin & VAH F RQRA TVF LVPRRHGK TWF LVP L I AL A L A T F - -KG IK I GYTAH IRKATEPVFEE I VARL RQWFGGER VDHVKGE - - \\
\hline Equid_her & IRHFRQRA TVFLVPRRHGK TWF L VPL I AL AL A T F - - KG IK I GYTAHIRKATEPVFDE I GARL RQWFGNSP \\
\hline & \\
\hline & - MRKER NGG IS T SGDF YF KAEARL QTHGH I I RVEFF \\
\hline virus & KVE Q I KK F NPN I T KHGQT YY Q T T CRWR E T DQ T V V I T F YKF D RE T P I I KQN T PY F S TND F R AK PY I CANA. \\
\hline :svirus_1 & - - E L VNK YKARKKL YKGF YY KA AHT P GKK D G A L VVSF FYKF T EAGRNIK T TD I PMAVN E F R S I VYKQKDTH \\
\hline & . \\
\hline virus 3 & 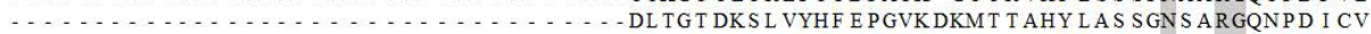 \\
\hline Ranic & $\ldots . .$. SLQG T AENL IYT S DPAVK AKP S T CHF MAS GSNS ARGQNPDL VI \\
\hline Rani & 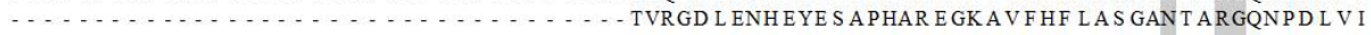 \\
\hline Muri & $\ldots \ldots \ldots$ VIL Y S HPGKK S S T LMCA TCF NKN S I RGQT F N L L F \\
\hline & ..... T I I F GPPNKKP S T LMCA TCF NKNS I R \\
\hline Human & $\ldots$. T I I Y TRPGGRSS S LMCA TCF NKNS I RGQT F NLLY \\
\hline Panine_he & - - VIS I DHRGAKS T AL F - A S CYNTNS I RGQNFH L L L \\
\hline Gallid_ & $\ldots$. TI T F T FRNGNKS A I VFA S SQNTNG L RGQDFNF L F \\
\hline & 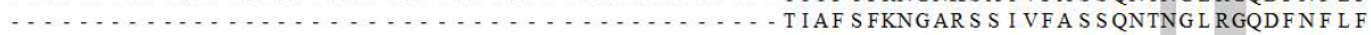 \\
\hline Meleagrid & ... - TITF SFPSGSRSTVTFASSHNTNS IRGQDFNLLF \\
\hline & 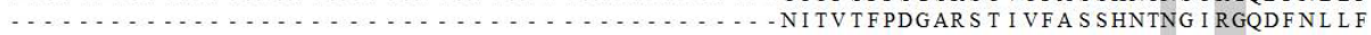 \\
\hline Maca & $\ldots . .$. T ISF SFPDG SRS T I VFA S SHNTNG I RGQDF NL L F \\
\hline Papine_her: & - - T I SF SFPDG SR S T I VFA S SHNTNG I RGQDF NL L F \\
\hline Cercopit & SF SFPDG SRST I VFA S SHNTNG I RGQDFNLLF \\
\hline Huma & - TISF S F PDG SR S T I VFA S SHNTNG I RGQDFNL L F \\
\hline Bovir & - . - VI SF S F PDGAR S T I VFA S SHNTNG I RGQDF NL L F \\
\hline Equid_herpesvirus_ $\overline{1}$ & - NISF SFPDG SKS T I VFA S SHNTNG I RGQDF NL L F \\
\hline
\end{tabular}

T4 Amphioxus abalone_herpesvirus Ostreid_herpesvirus_1 Ictalurid_herpesvirus_1 Cyprinid_herpesvirus_3 Ranid_herpesvirus_2 Ranid_herpesvirus_1 Murid_herpesvirus_4 Ovine herpesvirus 2 Human herpesvirus_8 Panine_herpesvirus_2 Gallid_herpesvirus_1 Psittacid_herpesvirus_1 Meleagrid herpesvirus Suid_herpesvinus_1 Macacine_herpesvirus_1 Papine_herpesvirus_2 Cercopithecine herpesvirus 2 Human_herpesvirus_1 Bovine herpesvirus 1 Equid_herpesvirus_1

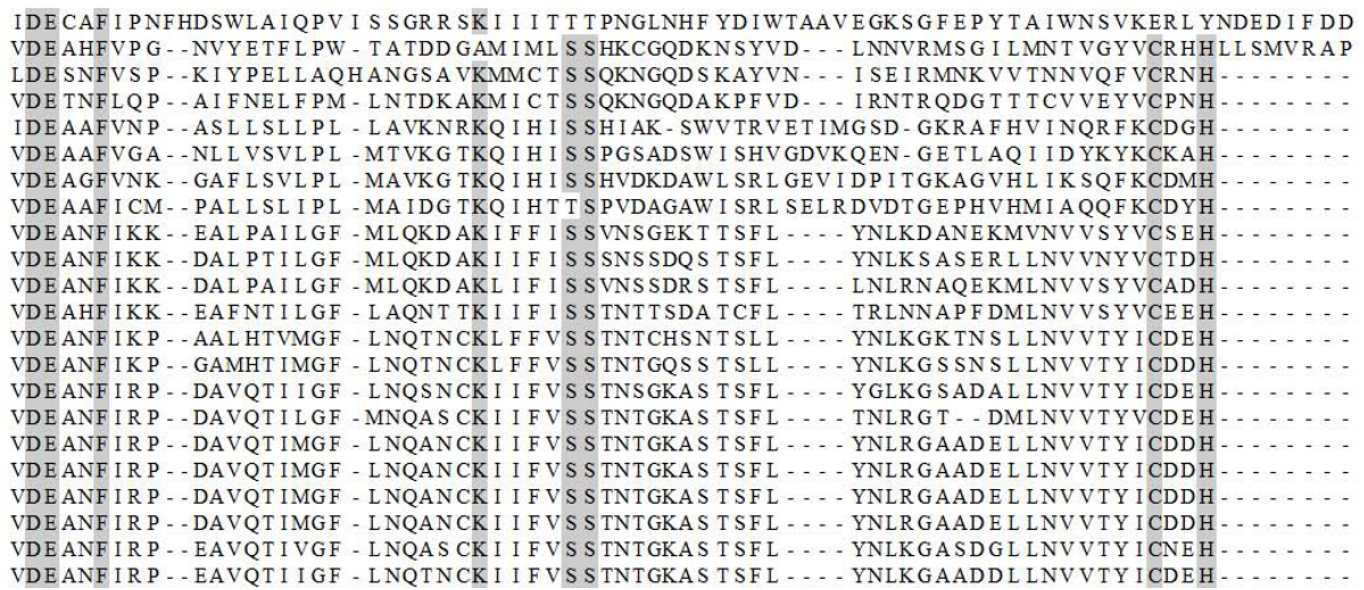

Figure 1 Alignment of ATP hydrolase domains from terminase protein sequences. ClustalW alignment of one of the conserved regions of the putative terminase gene - the ATP hydrolase (ATPase) domain from various herpesviruses taken from Table 3, identified using Interproscan. Grey background $=>90 \%$ conserved amino acids.

virus and the abalone and oyster viruses in a deep invertebrate clade. The level of divergence makes estimation of the relative divergence times of the 3 herpesvirus families difficult. Interestingly, the amphioxus virus is in the clade with mollusc viruses, which may not have been expected given the amphioxus chordate host lineage is more aligned with vertebrates than molluscs.

The invertebrate herpesvirus clade provides a unique branching point to inform the metazoan diversification of the herpesviruses. It is thought that during the 


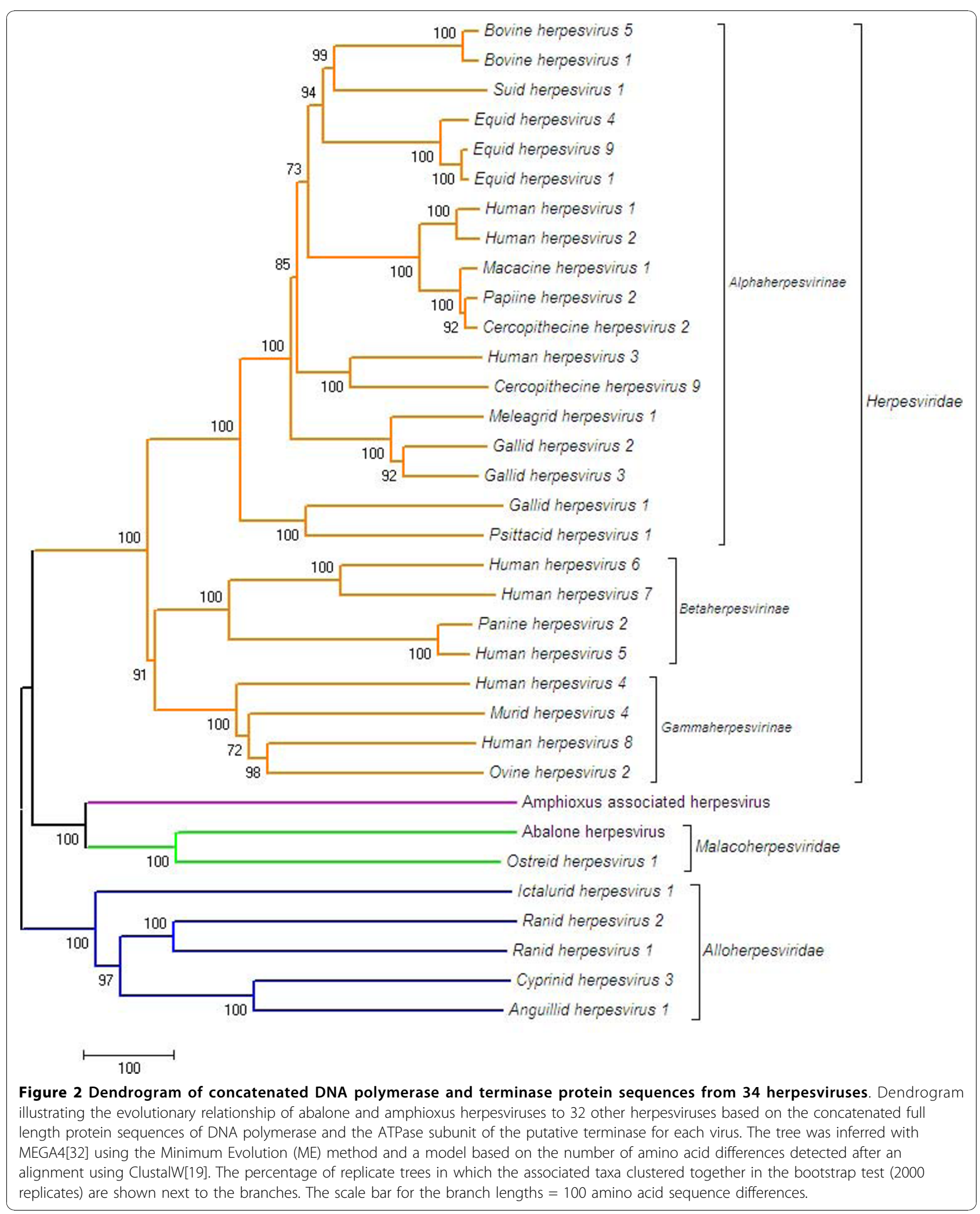


Table 2 Branchiostoma floridae (amphioxus) homologues of OsHV-1 coding sequences

\begin{tabular}{|c|c|c|c|c|c|}
\hline \multirow{2}{*}{$\begin{array}{c}\text { OsHV-1 } \\
\text { Accession/ORF }\end{array}$} & \multicolumn{2}{|c|}{ Branchiostoma floridae } & \multicolumn{3}{|c|}{ BLASTP result } \\
\hline & Accession & Location & Ident. & Score & E value \\
\hline $\begin{array}{l}\text { YP_024639.1 ORF100 } \\
\text { DNA polymerase }\end{array}$ & $\begin{array}{l}\text { XP_002591163.1 } \\
\text { DNA polymerase }\end{array}$ & $\begin{array}{c}55013 . .60373 \\
\text { no introns }\end{array}$ & $28 \%$ & 379 & e-102 \\
\hline YP_024567.1 ORF22 & XP_002591166.1 & $\begin{array}{c}67372.72375 \\
\text { no introns }\end{array}$ & $24 \%$ & 128 & $8 e-27$ \\
\hline $\begin{array}{c}\text { YP_024552.1 } \\
\text { YP_024593.1 } \\
\text { ORF7, ORF49 family primase-helicase }\end{array}$ & XP_002591168.1 & $\begin{array}{l}76325.79696 \\
\text { no introns }\end{array}$ & $24 \%$ & 122 & $3 e-25$ \\
\hline YP_024630.1 ORF91 & XP_002591169.1 & $\begin{array}{c}80230 . .85225 \\
\text { introns predicted }\end{array}$ & $29 \%$ & 114 & $2 e-23$ \\
\hline YP_024606.1 ORF66 AE_Prim_S_like primase & XP_002591170.1 & $\begin{array}{c}86185 . .88995 \\
\text { no introns }\end{array}$ & $24 \%$ & 239 & $2 e-60$ \\
\hline YP_024573.1 ORF28 & XP_002591172.1 & $\begin{array}{c}94244.96667 \\
\text { no introns }\end{array}$ & $22 \%$ & 107 & $4 \mathrm{e}-21$ \\
\hline YP_024641.1 ORF102 & XP_002591174.1 & $\begin{array}{l}99529 . .101919 \\
\text { no introns }\end{array}$ & $20 \%$ & 78 & $4 e-12$ \\
\hline YP_024645.1 ORF107 & $\begin{array}{c}\text { XP_002591175.1 } \\
\text { contains PAT1 } \\
\text { domain } \\
\text { pfam09770 }\end{array}$ & $\begin{array}{l}103007 . .105292 \\
\text { no introns }\end{array}$ & $24 \%$ & 65 & $2 \mathrm{e}-08$ \\
\hline YP_024584.1 ORF40 & XP_002591176.1 & $\begin{array}{c}105441 . .107045 \\
\text { no introns }\end{array}$ & $29 \%$ & 180 & $4 e-43$ \\
\hline YP_024643.1 ORF104 & XP_002591178.1 & $\begin{array}{c}108452 . .110641 \\
\text { no introns }\end{array}$ & $19 \%$ & 101 & $4 e-19$ \\
\hline YP_024615.1 ORF76 & XP_002591179.1 & $\begin{array}{c}112401 . .114281 \\
\text { no introns }\end{array}$ & $26 \%$ & 71 & $4 e-10$ \\
\hline YP_024624.1 ORF85 & XP_002591189.1 & $\begin{array}{l}137878 . .148379 \\
\text { introns predicted }\end{array}$ & $22 \%$ & 70 & 1e-09 \\
\hline $\begin{array}{c}\text { YP_024597.1 } \\
\text { YP_024608.1 } \\
\text { ORF54, ORF68 family membrane glycoprotein }\end{array}$ & $\begin{array}{l}\text { XP_002591190.1 } \\
\text { XP_002591197.1 } \\
\text { (possible gene } \\
\text { family) }\end{array}$ & $\begin{array}{c}148508.150751 \\
174789 . .176912 \text { no introns }\end{array}$ & $30 \%$ & 332 & $1 e-88$ \\
\hline YP_024591.1 ORF47 & XP_002591194.1 & $\begin{array}{c}163504.167571 \\
\text { no introns }\end{array}$ & $23 \%$ & 275 & $4 \mathrm{e}-71$ \\
\hline YP_024647.1 ORF109 terminase & $\begin{array}{l}\text { XP_002591195.1 } \\
\text { terminase }\end{array}$ & $\begin{array}{l}168081 . .170354 \\
\text { no introns }\end{array}$ & $31 \%$ & 308 & $2 e-81$ \\
\hline YP_024650.1 ORF112 & XP_002591198.1 & $\begin{array}{l}177489.179961 \\
\text { introns predicted }\end{array}$ & $23 \%$ & 68 & $2 e-09$ \\
\hline YP_024609.1 ORF69 & XP_002591200.1 & $\begin{array}{c}187709 . .188944 \\
\text { no introns }\end{array}$ & $25 \%$ & 80 & $3 e-13$ \\
\hline YP_024600.1 ORF57 & $\begin{array}{l}\text { XP_002610653.1 } \\
\text { chloride channel }\end{array}$ & $\begin{array}{l}\text { BRAFLscaffold_25 2304811..2311488 } \\
\text { introns predicted }\end{array}$ & $30 \%$ & 86 & $4 e-15$ \\
\hline
\end{tabular}

Note: $B$. floridae OsHV homologue locations are all on scaffold BRAFLscaffold_217, except for OsHV ORF57. All OsHV and B. floridae predicted proteins listed are of unknown function unless stated otherwise. Four $B$. floridae genes are predicted to contain introns. Also 4 other $B$. floridae genes in the scaffold BRAFLscaffold_217 between $60 \mathrm{~kb}$ and $150 \mathrm{~kb}$ encode proteins similar to apoptosis regulators like IAP-3 often present in herpesvirus genomes (not listed and not detected using OsHV sequences).

Cambrian era, the Bilaterial species diverged to generate the Protostomes (evolving into such animals as flatworms, molluscs and arthropods) and the Deuterostomes (from which the chordates and then the vertebrates evolved)[20,21]. Molluscs emerged more than 100 My before vertebrates with a bony skeleton (the current known range of herpesviruses in vertebrates). One hypothesis to explain the diversity of viruses within vertebrates and the positioning of the mollusc viruses among them, rather than as an ancestral outgroup, is the existence of diverse herpesviruses in Cambrian metazoans. Consistent with this hypothesis, previous estimates for the divergence of just the Herpesviridae in vertebrates indicate a divergence of alpha-, beta- and gammaherpesviruses to over $400 \mathrm{Mya}$, and longer times are predicted for divergence of Alloherpesviridae and Malacoherpesviridae[7]. An alternate hypothesis to explain the branching of the 3 herpesvirus 
Table 3 Genbank Accessions of Herpesvirus Polymerase and Terminase protein sequences used for phylogenetic analysis

\begin{tabular}{|c|c|c|}
\hline Virus & Polymerase & Terminase \\
\hline Abalone_herpesvirus & ADJ95314.1 & ADJ95315.1 \\
\hline Amphioxus_associated_virus & XP_002591163.1 & XP_002591195.1 \\
\hline Anguillid_herpesvirus_1 & YP_003358194.1 & YP_003358149.1 \\
\hline Bovine_herpesvirus_1 & NP_045328.1 & NP_045342.1 \\
\hline Bovine_herpesvirus_5 & NP_954917.1 & NP_954931.1 \\
\hline Cercopithecine_herpesvirus_2 & YP_164473.1 & YP_164457.1 \\
\hline Cercopithecine_herpesvirus_9 & NP_077443.1 & NP_077457.1 \\
\hline Cyprinid_herpesvirus_3 & YP_001096114.1 & YP_001096069.1 \\
\hline Equid_herpesvirus_1 & YP_053075.1 & YP_053090.1 \\
\hline Equid_herpesvirus_4 & NP_045247.1 & NP_045262.1 \\
\hline Equid_herpesvirus_9 & YP_002333511.1 & YP_002333526.2 \\
\hline Gallid_herpesvirus_1 & YP_182359.1 & YP_182378.2 \\
\hline Gallid_herpesvirus_2 & AAF66765.1 & YP_001033943.1 \\
\hline Gallid_herpesvirus_3 & NP_066862.1 & NP_066845.1 \\
\hline Human_herpesvirus_1 & NP_044632.1 & NP_044616.1 \\
\hline Human_herpesvirus_2 & P07918.1 & NP_044484.1 \\
\hline Human_herpesvirus_3 & NP_040151.1 & NP_040165.1 \\
\hline Human_herpesvirus_4 & YP_401712.1 & YP_401690.1 \\
\hline Human_herpesvirus_5 & P08546.2 & P16732.1 \\
\hline Human_herpesvirus_6 & NP_042931.1 & NP_042953.2 \\
\hline Human_herpesvirus_7 & P52342.1 & YP_073802.1 \\
\hline Human_herpesvirus_8 & AAC57086.1 & YP_001129382.1 \\
\hline Ictalurid_herpesvirus_1 & NP_041148.2 & NP_041153.2 \\
\hline Macacine_herpesvirus_1 & NP_851890.1 & NP_851874.1 \\
\hline Meleagrid_herpesvirus_1 & NP_073324.1 & NP_073308.1 \\
\hline Murid_herpesvirus_4 & NP_044849.1 & NP_044866.2 \\
\hline Ostreid_herpesvirus_1 & YP_024639.1 & YP_024647.1 \\
\hline Ovine_herpesvirus_2 & YP_438136.1 & YP_438152.1 \\
\hline Panine_herpesvirus_2 & NP_612698.1 & NP_612722.1 \\
\hline Papiine_herpesvirus_2 & YP_443877.1 & YP_443861.1 \\
\hline Psittacid_herpesvirus_1 & NP_944403.1 & NP_944422.2 \\
\hline Ranid_herpesvirus_1 & YP_656727.1 & YP_656697.1 \\
\hline Ranid_herpesvirus_2 & YP_656618.1 & YP_656576.1 \\
\hline Suid_herpesvirus_1 & YP_068333.1 & YP_068358.1 \\
\hline
\end{tabular}

families is that molluscs acquired herpesviruses by transmission in the aquatic environment, for example through association such as mollusc predation of early chordates. It appears that modern Malacoherpesviridae may have the ability to infect across species, a feature not typically observed in vertebrate herpesviruses, although the infection observed is restricted to related mollusc species[22].

As more sequence data and gene structure for Alloherpesviridae, Malacoherpesviridae and other invertebrate herpesviruses become available it will allow a more informative analysis of their evolution. Of particular interest will be new herpesviruses yet to be discovered in species which share bilateral symmetry such as amphioxus, sea squirts, flatworms or squid. Our discovery of clustered intact herpesvirus genes in amphioxus suggests an opportunistic integration has occurred in the amphioxus genome. This may not be a normal feature of infection and latency, but herpesviruses can occasionally integrate into the genome of their host[23]. Surprisingly, the nearest relatives of this chordate virus seem to be the viruses infecting molluscs rather than those of fish or frogs. Although herpesvirus particles have not been seen in the more primitive metazoan species, their existence is suspected; short herpes-like DNA sequences having been found in a metagenomic study of Hawaian coral[24]. Further metagenomic approaches similar to those described previously[25] and PCR-directed approaches[26] based on new sequences described here will enable these evolutionary questions to be addressed. The sequence information is also crucial for the development of molecular diagnostic tools to monitor and manage disease outbreaks.

The neurotropism of certain herpesviruses is well documented but this behaviour is not known outside the families of herpesviruses infecting terrestrial vertebrates $[27,28]$. The neurotropic tissue infection profile of the new gastropod virus analysed here is shared with some viruses within the Herpesviridae family. Convergent evolution may have given rise to the neurotropism seen in some members of the Herpesviridae and now the Malacoherpesviridae families. The rooting of a neurotropic invertebrate virus near or before the divergence of alpha-, beta-, and gammaherpesviruses, may also suggest that early mammalian herpesvirus precursors were neurotropic and that some have retained this feature over time. It is interesting to speculate as to the earliest functional interactions between sensory cells and viruses, as the first sign of neurons appeared over 600 million years ago in "cnidarians," (eg: hydra), but organisms basal to them like sponges do not have neurons or synapses[29]. Recent evidence indicates sponges have gene networks in cells which were precursors to nerve cells including proteins related to virus nerve entry receptors[30]. Others[24] have speculated on a link between herpesvirus neurotropism and the evolution of modern herpesviruses from ancestors infecting invertebrates such as Cnidaria (for example, coral or sea anemones), thought to be related to the first species with sensory receptors[31]. Further, the discovery reported here of a putative herpesvirus integrated into the genome of amphioxus hints at a wide diversity of herpesviruses within the invertebrate community, perhaps dating back to before the divergence of arthropods, molluscs and chordates. 
It will be exciting to discover such invertebrate herpesviruses and explore their links to ancient herpesvirus ancestors.

To accommodate the new abalone virus, which we have suggested naming abalone herpesvirus or AbHV-1, within the Herpesvirales order, we suggest the creation of a new genus called Haliotivirus within the Malacoherpesviridae family and assignment of AbHV-1 as a species under Haliotivirus (as Haliotid herpesvirus 1).

We have referred to the putative virus genome integrated into the Branchiostomid species chromosome as amphioxus-associated virus, AaHV-1. We suggest the species name Branchiostomid herpesvirus 1. Given the unique nature of the virus revealed by phylogenetic analysis and the unique evolutionary positioning of amphioxus as an invertebrate chordate, we suggest this virus, if classified, could be a member of a new family, Aspondyloherpesviridae (from the Greek for "no spine").

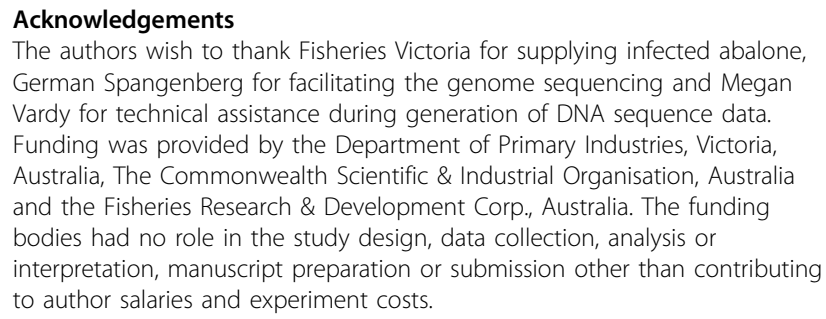

\section{Acknowledgements}

The authors wish to thank Fisheries Victoria for supplying infected abalone, German Spangenberg for facilitating the genome sequencing and Megan Vardy for technical assistance during generation of DNA sequence data. Funding was provided by the Department of Primary Industries, Victoria, Australia, The Commonwealth Scientific \& Industrial Organisation, Australia and the Fisheries Research \& Development Corp., Australia. The funding bodies had no role in the study design, data collection, analysis or interpretation, manuscript preparation or submission other than contributing to author salaries and experiment costs.

\section{Author details}

'Biosciences Research Division, Department of Primary Industries, 1 Park Drive, Bundoora, Victoria 3083, Australia. ${ }^{2}$ Biosciences Research Division, Department of Primary Industries, 475 Mickleham Road, Attwood Victoria 3049, Australia. ${ }^{3}$ Australian Animal Health Laboratory, CSIRO Livestock Industries, Geelong, VIC 3220, Australia. ${ }^{4}$ School of Plant Biology, University of Western Australia, 35 Stirling Hwy Crawley, W.A 6009, Australia. ${ }^{5}$ La Trobe University, Bundoora, Victoria 3086, Australia.

\section{Authors' contributions}

KWS, FW, BGC, SW conceived and designed the experiments; FW, NC performed the experiments; KWS, TS, DS analyzed the data; FW, SW, TS, DS, NC contributed reagents, materials, analysis tools; KWS, BGC wrote the paper. All authors have contributed to the editing or revision of the manuscript and approve its publication.

\section{Competing interests}

The authors declare that they have no competing interests.

Received: 4 August 2010 Accepted: 10 November 2010 Published: 10 November 2010

\section{References}

1. Hooper C, Hardy-Smith P, Handlinger J: Ganglioneuritis causing high mortalities in farmed Australian abalone (Haliotis laevigata and Haliotis rubra). Australian Veterinary Journal 2007, 85:188-193.

2. NACA: Quarterly Aquatic Animal Disease Report (Asia and Pacific Region) 2006/1. Book Quarterly Aquatic Animal Disease Report (Asia and Pacific Region) 2006/1 (Editor ed.^eds.). City 2006, 5.

3. Tan J, Lancaster M, Hyatt A, van Driel R, Wong F, Warner S: Purification of a herpes-like virus from abalone (Haliotis spp.) with ganglioneuritis and detection by transmission electron microscopy. Journal of Virological Methods 2008, 149:338-341.
4. Chang PH, Kuo ST, Lai SH, Yang HS, Ting YY, Hsu CL, Chen HC: Herpes-like virus infection causing mortality of cultured abalone Haliotis diversicolor supertexta in Taiwan. Dis Aquat Organ 2005, 65:23-27.

5. Corbeil S, Colling A, Williams LM, Wong FYK, Savin K, Warner S, Murdoch B, Cogan NOI, Sawbridge Tl, Fegan M, et al: Development and validation of a TaqMan PCR assay for the Australian abalone herpes-like virus. Dis Aquat Organ 2010, 91:1-10.

6. Davison AJ, Eberle R, Ehlers B, Hayward GS, McGeoch DJ, Minson AC, Pellett PE, Roizman B, Studdert MJ, Thiry E: The order Herpesvirales. Arch Virol 2009, 154:171-177.

7. McGeoch DJ, Rixon FJ, Davison AJ: Topics in herpesvirus genomics and evolution. Virus Research 2006, 117:90-104

8. Waltzek TB, Kelley GO, Alfaro ME, Kurobe T, Davison AJ, Hedrick RP: Phylogenetic relationships in the family Alloherpesviridae. Dis Aquat Organ 2009, 84:179-194.

9. Le Deuff RM, Renault T: Purification and partial genome characterization of a herpes-like virus infecting the Japanese oyster, Crassostrea gigas. J Gen Virol 1999, 80:1317-1322.

10. Silander K, Saarela J: Whole genome amplification with Phi29 DNA polymerase to enable genetic or genomic analysis of samples of low DNA yield. Methods Mol Biol 2008, 439:1-18.

11. Rutherford K, Parkhill J, Crook J, Horsnell T, Rice P, Rajandream MA, Barrell B: Artemis: sequence visualization and annotation. Bioinformatics 2000 16:944-945.

12. Farley CA, Banfield WG, Kasnic G Jr, Foster WS: Oyster herpes-type virus. Science 1972, 178:759-760.

13. Davison AJ, Trus BL, Cheng N, Steven AC, Watson MS, Cunningham C, Le Deuff RM, Renault T: A novel class of herpesvirus with bivalve hosts. J Gen Virol 2005, 86:41-53.

14. Altschul SF, Madden TL, Schaffer AA, Zhang J, Zhang Z, Miller W, Lipman DJ: Gapped BLAST and PSI-BLAST: a new generation of protein database search programs. Nucleic Acids Res 1997, 25:3389-3402.

15. Putnam NH, Butts T, Ferrier DE, Furlong RF, Hellsten U, Kawashima T, Robinson-Rechavi M, Shoguchi E, Terry A, Yu JK, et al: The amphioxus genome and the evolution of the chordate karyotype. Nature 2008, 453:1064-1071.

16. Davison AJ: Channel catfish virus: a new type of herpesvirus. Virology 1992, 186:9-14

17. Yang K, Homa F, Baines JD: Putative terminase subunits of herpes simplex virus 1 form a complex in the cytoplasm and interact with portal protein in the nucleus. J Virol 2007, 81:6419-6433.

18. Zdobnov E, Apweiler R: InterProScan - an integration platform for the signature-recognition methods in InterPro. Bioinformatics 2001, 17:847-848.

19. Thompson JD, Higgins DG, Gibson TJ: CLUSTAL W: improving the sensitivity of progressive multiple sequence alignment through sequence weighting, position-specific gap penalties and weight matrix choice. Nucleic Acids Res 1994, 22:4673-4680

20. Lartillot N, Philippe $\mathrm{H}$ : Improvement of molecular phylogenetic inference and the phylogeny of Bilateria. Philos Trans R Soc Lond B Biol Sci 2008, 363:1463-1472.

21. Nielsen C: Six major steps in animal evolution: are we derived sponge larvae? Evol Dev 2008, 10:241-257.

22. Arzul I, Renault T, Lipart C, Davison AJ: Evidence for interspecies transmission of oyster herpesvirus in marine bivalves. J Gen Virol 2001, 82:865-870.

23. Arbuckle JH, Medveczky MM, Luka J, Hadley SH, Luegmayr A, Ablashi D, Lund TC, Tolar J, De Meirleir K, Montoya JG, et al: The latent human herpesvirus-6A genome specifically integrates in telomeres of human chromosomes in vivo and in vitro. Proc Natl Acad Sci USA 2010, 107:5563-5568.

24. Vega Thurber RL, Barott KL, Hall D, Liu H, Rodriguez-Mueller B, Desnues C, Edwards RA, Haynes M, Angly FE, Wegley L, Rohwer FL: Metagenomic analysis indicates that stressors induce production of herpes-like viruses in the coral Porites compressa. Proc Natl Acad Sci USA 2008, 105:18413-18418.

25. Suttle CA: Viruses in the sea. Nature 2005, 437:356-361.

26. Ehlers B, Dural G, Yasmum N, Lembo T, de Thoisy B, Ryser-Degiorgis MP, Ulrich RG, McGeoch DJ: Novel mammalian herpesviruses and lineages within the Gammaherpesvirinae: cospeciation and interspecies transfer. J Virol 2008, 82:3509-3516. 
27. Enquist LW, Husak PJ, Banfield BW, Smith GA: Infection and spread of alphaherpesviruses in the nervous system. Adv Virus Res 1998, 51:237-347.

28. Terry LA, Stewart JP, Nash AA, Fazakerley JK: Murine gammaherpesvirus-68 infection of and persistence in the central nervous system. $J$ Gen Virol 2000, 81:2635-2643.

29. Sakarya O, Armstrong KA, Adamska M, Adamski M, Wang IF, Tidor B, Degnan BM, Oakley TH, Kosik KS: A post-synaptic scaffold at the origin of the animal kingdom. PLoS One 2007, 2:e506.

30. Richards GS, Simionato E, Perron M, Adamska M, Vervoort M, Degnan BM: Sponge genes provide new insight into the evolutionary origin of the neurogenic circuit. Curr Biol 2008, 18:1156-1161.

31. Putnam NH, Srivastava M, Hellsten U, Dirks B, Chapman J, Salamov A, Terry A, Shapiro H, Lindquist E, Kapitonov W, et al: Sea anemone genome reveals ancestral eumetazoan gene repertoire and genomic organization. Science 2007, 317:86-94.

32. Tamura K, Dudley J, Nei M, Kumar S: MEGA4: Molecular Evolutionary Genetics Analysis (MEGA) software version 4.0. Mol Biol Evol 2007, 24:1596-1599.

doi:10.1186/1743-422X-7-308

Cite this article as: Savin et al.: A neurotropic herpesvirus infecting the gastropod, abalone, shares ancestry with oyster herpesvirus and a herpesvirus associated with the amphioxus genome. Virology Journal 2010 7:308

\section{Submit your next manuscript to BioMed Central} and take full advantage of:

- Convenient online submission

- Thorough peer review

- No space constraints or color figure charges

- Immediate publication on acceptance

- Inclusion in PubMed, CAS, Scopus and Google Scholar

- Research which is freely available for redistribution

Submit your manuscript at www.biomedcentral.com/submit 